\title{
Saturniidae and Sphingidae (Lepidoptera, Bombycoidea) assemblage in Vossoroca, Tijucas do Sul, Paraná, Brazil
}

\author{
FÁBIO L. SANTOS, MIRNA M. CASAGRANDE and OLAF H.H. MIELKE
}

Universidade Federal do Paraná, Departamento de Zoologia, Laboratório de Estudos de Lepidoptera Neotropical, Caixa Postal 19020, 81531-980 Curitiba, PR, Brasil

Manuscript received on July 14, 2014; accepted for publication on October 24, 2014

\begin{abstract}
The richness and abundance of the assemblage of species in a community are a way to understand the patterns of diversity found around the world. This study describes the assemblages of Saturniidae and Sphingidae in an area of Montana Mixed Ombrophilous Forest, Tijucas do Sul, Paraná, Brazil, 880m, $25^{\circ} 50^{\prime} 8.93^{\prime \prime S}, 49^{\circ} 02^{\prime} 55.20^{\prime \prime W}$. Samples were collected between November 2010 and September 2011 during two nights at the twelve new moons periods with a light trap equipped with black light lamp (ultraviolet) of 46 watts between two mercury mixed-light lamps HWL 250 watts from 6:00 p.m to 06:00 a.m. The sampling effort totaled 288 hours. This sampling effort was responsible for the capture of 1235 specimens, distributed in 124 species: 858 specimens and 86 species of Saturniidae, and 377 specimens and 38 species of Sphingidae. It is noteworthy the sampling of 10 new species: one of Automerella Michener, 1949, two of Hylesia Hübner, [1820], one of Lonomia Walker, 1855 and six of Periga Walker, 1855, that will be described.
\end{abstract}

Key words: Neotropical, survey, moths, community.

\section{INTRODUCTION}

Faunistic studies allow us to answer primary fundamental questions in ecology, such as "what is the species composition in a given locality?" (Silveira et al. 2010), where species richness is commonly used as a community descriptor in many ecological studies (Magurran 2004).

All the information provided by these studies allow us to make long-term predictions about environmental changes, understand the resilience of organisms and their persistence in small fragmented habitats, which are isolated and / or have a long history of disturbance (Rubinoff and Powell 2004).

Correspondence to: Fábio Luis dos Santos

E-mail: flstche@yahoo.com.br
Without faunistic research performed in a systematic and comparative way, it is difficult to understand the real patterns of distribution of species richness, between different areas used by their populations and how this richness is distributed among different spatial and temporal niches. Likewise, it is difficult to assess the efficiency of collection methods. Furthermore, it also limits more robust conclusions regarding the use of these organisms as bioindicators (Carneiro and Leivas 2012), and support to conservation, preservation, environmental education and management strategies (i.e. Santos et al. 2008, Silveira et al. 2010, Freitas et al. 2011).

Studies on Saturniidae and Sphingidae are still scarce, even though they belong to a groups with 
a broad geographical distribution and reasonable taxonomic resolution (Lemaire 1978, 1980, 1988, 2002, Kitching and Cadiou 2000). As one example, the state of Paraná, despite having a track record of studies and support for research on Lepidoptera, still has a few lists: Saturniidae (Marinoni et al. 1999) and Sphingidae (Laroca and Mielke 1975, Laroca et al. 1989, Marinoni et al. 1997).

These moths, mostly nocturnal, are greatly diversified and ecologically important. They belong to the folivorous guild, having considerable influence on ecosystems due to being herbivorous and their role in the conversion of biomass (New 2013). It is estimated that food webs based on a plantherbivore relationship include a large proportion of the biodiversity, somewhere around $75 \%$ (Price 2002). They are equally important as prey within the food chains of birds, bats, parasitoids and parasites. Particularly only Sphingidae are important as pollinators once the Saturniidae species do not feed as adults (Janzen 1984).

Therefore, they represent important potential indicators of ecosystem health (Highland et al. 2013), has charismatic flagship species for conservation campaigns (New 2013), and their knowledge is important for understanding the complexity of tropical biodiversity and its maintenance (Balvanera et al. 2002, Novotny et al. 2010).

The area chosen for this research was the locality of Vossoroca, Tijucas do Sul, Paraná, within the Atlantic Forest biome, one of the global "hotspots" (Myers et al. 2000, Lewinsohn et al. 2005). This is the most altered Brazilian biome due to anthropogenic pressure (Mittermeier et al. 1998, Galindo-Leal and Câmara 2005).

Within this biome, the Montana Mixed Ombrophilous Forest phytoecological region, which historically covered an area equivalent to $164,042.75$ $\mathrm{km}^{2}$ (Hirota 2005) and extended from the southern part of state of São Paulo to the Misiones Province of Argentina (IBGE 1992), currently covers about 12\% of its original distribution (Ribeiro et al. 2009).
Considering the population growth, the proximity to large urban centers, the inadequate land management and the location of one of the most important dams (Vossoroca Dam), it is critical to know and monitor this region in order to preserve the local biodiversity and the value of its ecosystem services.

This study was aimed at increasing our knowledge about the Saturniidae and Sphingidae assemblage within the Montana Mixed Ombrophilous Forest, thus contributing towards our understanding of the diversity of the Atlantic Forest biome.

\section{MATERIALS AND METHODS}

STUDY AREA

The study was conducted at the farm of the "Associação dos Professores da Universidade Federal do Paraná" located in Vossoroca, Tijucas do Sul, Paraná, Brazil (2550'08.93"S, 4902'55.20"W), at an altitude of $880 \mathrm{~m}$. This study site is located within the "Primeiro Planalto Paranaense", of the "Planalto de Curitiba" subunit, characterized by an altitudinal gradient ranging between 560 and $1240 \mathrm{~m}$ with the predominance of elongated and flattened plateaus, convex slopes and "V" shaped valleys (Maack 1981, MINEROPAR 2001).

The climate is humid mesothermal subtropical or $\mathrm{Cfb}$ (according to the Wilhelm Koppen climate classification), with cool summers and no occurrence of severe and frequent frosts, probably due to high cloud cover present almost year round. The mean temperatures of the warmest months are below $22^{\circ} \mathrm{C}$ and the coldest months are lower than $18^{\circ} \mathrm{C}$. The average annual rainfall is $1400 \mathrm{~mm}$, with a concentration of rainfall during the period from October to March, without a defined dry season (INMET 2009).

Currently, the surrounding areas are dominated by agricultural activities, pasture and mostly reforestations using exotic species belonging to logging companies, altering the natural vegetation cover. However, the study area is part of a fragment of native forest within larger reminiscent forests 
which are in different stages of succession, interspersed with civilian constructions and small farms. It is also part of the APA ("Área de Proteção Ambiental”) of Guaratuba (IAP 2003).

Both local and regional floristic and phytosociological sources (i.e. Rondon-Neto et al. 2002, Liebsch and Acra 2004, Cordeiro and Rodrigues 2007, Sonego et al. 2007) can be consulted to better understand the type, composition and structure of the vegetation in the study area in the context of Montana Mixed Ombrophilous Forest.

\section{SAMPLING}

The collections were conducted on twelve excursions during the new moons, between November 2010 and September 2011, sampling between 06:00 p.m. and 06:00 a.m. hours during two nights, for a total of 24 nights and 288 hours.

The luminous attraction constituted of a reflective white cloth sheet, five meters long by three meters high, with a 46 watt black light (ultraviolet) Golden ${ }^{\circledR}$ lamp centrally positioned in front, along with two 250 watt mercury vapor mixed light HWL Osram ${ }^{\circledR}$ lamps suspended $1.5 \mathrm{~m}$ above the ground and $1.5 \mathrm{~m}$ apart from one another.

Specimens were collected manually and directly from the sheet or using an entomological net. Each specimen was then sacrificed with a drop of ammonia injected into the thorax, or placed inside a killing jar containing ammonia or ether, and then packaged in individual entomological envelopes with the collection time information. Continuous searches were also carried out within a $10 \mathrm{~m}$ radius in order to collect specimens that land on the vegetation or soil near the light. This method has the advantage of allowing the collection of undamaged specimens, preserving important characteristics used for identification and for future taxonomical studies (Axmacher and Fiedler 2004).

In the laboratory, the collected specimens were sexed, individually numbered and identified, and their date was entered in a database. Some of the specimens were prepared and deposited in the Coleção Entomológica Pe. Jesus Santiago Moure (DZUP), Departamento de Zoologia, Universidade Federal do Paraná, Curitiba, Paraná, Brazil. Others were dried, placed in envelopes and stored in the DZUP entomological collection.

The identifications were made by comparing then with identified specimens in the DZUP collection, by consulting specialists or through the following publications: for Saturniidae (D'Abrera 1995, Lemaire 1978, 1980, 1988, 2002) and for Sphingidae (D'Abrera 1986, Kitching and Cadiou 2000, Martin et al. 2011).

Minet (1994), Heppner (1996), Lemaire (1978, 1980, 1988, 2002), Balcázar-Lara and Wolfe (1997) (Ceratocampinae), Lemaire and Minet (1998), Camargo et al. (2009) (Arsenurinae), and the molecular phylogenies of Regier et al. (2008a, b) were used for the taxonomy of Saturniidae. For Sphingidae, the taxonomy was based on Kitching and Cadiou (2000), observing the relationships proposed by Kawahara et al. (2009).

\section{DATA ANALYSIS}

The richness and dominance were chosen to describe and characterize the structure of the assemblage that was also qualitatively compared with those from other localities for which there are published inventories. These lists of species were updated to reflect the most recent changes in the taxonomy of the species. Places with more than 10 species were chosen, in order to represent the neighboring areas (Table I). These areas are from the Brazilian states of Espirito Santo, Mato Grosso do Sul, Minas Gerais, Paraná, Rio de Janeiro, Rio Grande do Sul, São Paulo and Santa Catarina, and also from Misiones in Argentina.

Following Brose and Martinez (2004), Jackknife 2 was calculated in order to evaluate the sampling efficiency, to generate an estimated richness with the most appropriate richness estimator and in order to prepare of rarefaction 
curves. The $\mathrm{X}$ axis was transformed for individuals following Gotelli and Colwell (2001). Both procedures were implemented with the use of the EstimateS 8.2 program (Colwell 2005).

\section{RESULTS}

With 288 hours of sampling effort, 1,235 specimens $(\mathrm{N})$ were collected, distributed in 124 species (S), with 858 specimens representing

TABLE I

Checklist of the localities with inventories that were used to compare with the fauna of Tijucas do Sul, Vossoroca, Paraná, Brazil.

\begin{tabular}{|c|c|c|c|c|c|c|}
\hline Locality & State & Reference & Family** & Altitude & Latitude & Longitude \\
\hline $\begin{array}{l}\text { Belo Horizonte } \\
\text { (MG) }\end{array}$ & Minas Gerais & Camargo and Becker (1999) & SAT & $858 \mathrm{~m}$ & $19^{\circ} 56^{\prime} \mathrm{s}$ & $43^{\circ} 56^{\prime} \mathrm{w}$ \\
\hline $\begin{array}{l}\text { Bodoquena } \\
\text { (MS) }\end{array}$ & Mato Grosso do Sul & Camargo and Becker (1999) & SAT & $132 \mathrm{~m}$ & $20^{\circ} 32^{\prime} \mathrm{s}$ & $56^{\circ} 42^{\prime} w$ \\
\hline Lages (SC) & Santa Catarina & Siewert et al. (2010) & SAT & $916 \mathrm{~m}$ & $27^{\circ} 48^{\prime} 57^{\prime \prime} \mathrm{s}$ & $50^{\circ} 19^{\prime} 33^{\prime \prime} \mathrm{w}$ \\
\hline $\begin{array}{l}\text { Nova Lima } \\
(\mathrm{MG})\end{array}$ & Minas Gerais & Camargo and Becker (1999) & SAT & $850 \mathrm{~m}$ & $20^{\circ} 00^{\prime} \mathrm{s}$ & $43^{\circ} 54^{\prime} w$ \\
\hline Paracatu (MG) & Mato Grosso & Camargo and Becker (1999) & SAT & $920 \mathrm{~m}$ & $17^{\circ} 13^{\prime} \mathrm{s}$ & $46^{\circ} 52^{\prime} w$ \\
\hline $\begin{array}{l}\text { Rio Brilhante } \\
\text { (MS) }\end{array}$ & Mato Grosso do Sul & Camargo and Becker (1999) & SAT & $400 \mathrm{~m}$ & $21^{\circ} 48 \mathrm{~s}$ & $54^{\circ} 32^{\prime} w$ \\
\hline $\begin{array}{l}\text { Rio Verde de } \\
\text { Mato Grosso } \\
\text { (MS) }\end{array}$ & Mato Grosso do Sul & Camargo and Becker (1999) & SAT & $330 \mathrm{~m}$ & $18^{\circ} 55^{\prime} \mathrm{s}$ & $54^{\circ} 50^{\prime} w$ \\
\hline Salesópolis (SP) & São Paulo & Duarte et al. (2008) & SAT & $900 \mathrm{~m}$ & $23^{\circ} 38^{\prime} \mathrm{s}$ & $45^{\circ} 52^{\prime} w$ \\
\hline Salobra (MS) & Mato Grosso do Sul & Camargo and Becker (1999) & SAT & $100 \mathrm{~m}$ & $20^{\circ} 15^{\prime} \mathrm{s}$ & $56^{\circ} 40^{\prime} \mathrm{w}$ \\
\hline $\begin{array}{l}\text { São Bento do } \\
\text { Sul (SC) }\end{array}$ & Santa Catarina & Siewert et al. (2010) & SAT & $838 \mathrm{~m}$ & $26^{\circ} 15^{\prime} 00^{\prime \prime} \mathrm{s}$ & $49^{\circ} 22^{\prime} 44^{\prime \prime} \mathrm{w}$ \\
\hline $\begin{array}{l}\text { São Francisco } \\
\text { de Paula (RS) }\end{array}$ & Rio Grande do Sul & $\begin{array}{l}\text { Nunes et al. }(2003,2004), \\
\text { Prestes et al. (2009) }\end{array}$ & SAT & $907 \mathrm{~m}$ & $29^{\circ} 26^{\prime} 52^{\prime \prime s}$ & $50^{\circ} 35^{\prime} 02^{\prime \prime} \mathrm{w}$ \\
\hline $\begin{array}{l}\text { Sete Lagoas } \\
\text { (MG) }\end{array}$ & Minas Gerais & Camargo and Becker (1999) & SAT & $761 \mathrm{~m}$ & $13^{\circ} 28^{\prime} \mathrm{s}$ & $44^{\circ} 15^{\prime} \mathrm{w}$ \\
\hline $\begin{array}{l}\text { Três Marias } \\
\text { (MG) }\end{array}$ & Minas Gerais & Camargo and Becker (1999) & SAT & $538 \mathrm{~m}$ & $18^{\circ} 12^{\prime} \mathrm{s}$ & $45^{\circ} 14^{\prime} \mathrm{w}$ \\
\hline Unaí (MG) & Minas Gerais & Camargo and Becker (1999) & SAT & $575 \mathrm{~m}$ & $16^{\circ} 21^{\prime} \mathrm{s}$ & $46^{\circ} 54^{\prime} w$ \\
\hline Urubici (SC) & Santa Catarina & Siewert et al. (2010) & SAT & $915 \mathrm{~m}$ & $28^{\circ} 00^{\prime} 54^{\prime \prime} \mathrm{s}$ & $49^{\circ} 35^{\prime} 31^{\prime \prime} w$ \\
\hline Antonina (PR) & Paraná & $\begin{array}{l}\text { Marinoni et al. (1997), } \\
\text { Marinoni et al. (1999) }\end{array}$ & SAT; SPH & $60 \mathrm{~m}$ & $25^{\circ} 28^{\prime} \mathrm{s}$ & $48^{\circ} 50^{\prime} w$ \\
\hline Blumenau (SC) & Santa Catarina & $\begin{array}{l}\text { Siewert et al. (2010), } \\
\text { Siewert and Silva (2010) }\end{array}$ & SAT; SPH & $21 \mathrm{~m}$ & $26^{\circ} 54^{\prime} 32^{\prime \prime s}$ & $49^{\circ} 04^{\prime} 20^{\prime \prime} \mathrm{w}$ \\
\hline Colombo (PR) & Paraná & $\begin{array}{l}\text { Marinoni et al. (1997), } \\
\text { Marinoni et al. (1999) }\end{array}$ & SAT; SPH & $915 \mathrm{~m}$ & $25^{\circ} 20^{\prime} \mathrm{s}$ & $49^{\circ} 14^{\prime} w$ \\
\hline Fênix (PR) & Paraná & $\begin{array}{l}\text { Marinoni et al. (1997), } \\
\text { Marinoni et al. (1999) }\end{array}$ & SAT; SPH & $350 \mathrm{~m}$ & $23^{\circ} 54^{\prime} \mathrm{s}$ & $51^{\circ} 58^{\prime} \mathrm{w}$ \\
\hline $\begin{array}{l}\text { Guarapuava } \\
\text { (PR) }\end{array}$ & Paraná & $\begin{array}{l}\text { Marinoni et al. (1997), } \\
\text { Marinoni et al. (1999) }\end{array}$ & SAT; SPH & $740 \mathrm{~m}$ & $25^{\circ} 40^{\prime} \mathrm{s}$ & $52^{\circ} 01^{\prime} w$ \\
\hline Joinville (SC) & Santa Catarina & $\begin{array}{l}\text { Siewert et al. (2010), } \\
\text { Siewert and Silva (2010) }\end{array}$ & SAT; SPH & $4,5 \mathrm{~m}$ & $26^{\circ} 18^{\prime} 14^{\prime \prime s}$ & $48^{\circ} 50^{\prime} 45^{\prime \prime} \mathrm{w}$ \\
\hline $\begin{array}{l}\text { Jundiaí do Sul } \\
\text { (SC) }\end{array}$ & Santa Catarina & $\begin{array}{l}\text { Marinoni et al. (1997), } \\
\text { Marinoni et al. (1999) }\end{array}$ & SAT; SPH & $500 \mathrm{~m}$ & $23^{\circ} 26^{\prime} \mathrm{s}$ & $50^{\circ} 16^{\prime} \mathrm{w}$ \\
\hline $\begin{array}{l}\text { Ponta Grossa } \\
\text { (PR) }\end{array}$ & Paraná & $\begin{array}{l}\text { Marinoni et al. (1997), } \\
\text { Marinoni et al. (1999) }\end{array}$ & SAT; SPH & $880 \mathrm{~m}$ & $25^{\circ} 14^{\prime} \mathrm{s}$ & $50^{\circ} 03^{\prime} \mathrm{w}$ \\
\hline $\begin{array}{l}\text { Santa Teresa } \\
\text { (ES) }\end{array}$ & Espírito Santo & Brown Jr and Freitas (1999) & SAT; SPH & $650 \mathrm{~m}$ & $19^{\circ} 56^{\prime} 10^{\prime \prime s}$ & $40^{\circ} 36^{\prime} 06^{\prime \prime} \mathrm{w}$ \\
\hline
\end{tabular}


TABLE I (continuation)

\begin{tabular}{|c|c|c|c|c|c|c|}
\hline Locality & State & Reference & Family** & Altitude & Latitude & Longitude \\
\hline $\begin{array}{l}\text { São José dos } \\
\text { Pinhais (PR) }\end{array}$ & Paraná & $\begin{array}{l}\text { Marinoni et al. (1997), } \\
\text { Marinoni et al. (1999) }\end{array}$ & SAT; SPH & $1050 \mathrm{~m}$ & $25^{\circ} 34^{\prime} \mathrm{s}$ & $49^{\circ} 01^{\prime} w$ \\
\hline Seara (SC) & Santa Catarina & $\begin{array}{l}\text { Siewert et al. (2010), Siewert } \\
\text { and Silva (2010) }\end{array}$ & SAT; SPH & $550 \mathrm{~m}$ & $27^{\circ} 08^{\prime} 56^{\prime \prime} \mathrm{s}$ & $52^{\circ} 18^{\prime} 39^{\prime \prime} w$ \\
\hline $\begin{array}{l}\text { Telêmaco Borba } \\
\text { (PR) }\end{array}$ & Paraná & $\begin{array}{l}\text { Marinoni et al. (1997), } \\
\text { Marinoni et al. (1999) }\end{array}$ & SAT; SPH & $750 \mathrm{~m}$ & $24^{\circ} 17^{\prime} \mathrm{s}$ & $50^{\circ} 37^{\prime} \mathrm{w}$ \\
\hline Brusque (SC) & Santa Catarina & Siewert and Silva (2010) & SPH & $21 \mathrm{~m}$ & $27^{\circ} 05^{\prime} 52^{\prime \prime} \mathrm{s}$ & $48^{\circ} 55^{\prime} 04^{\prime \prime} \mathrm{w}$ \\
\hline Itaiópolis (SC) & Santa Catarina & Siewert and Silva (2010) & SPH & $925 \mathrm{~m}$ & $26^{\circ} 20^{\prime} 09^{\prime \prime} \mathrm{s}$ & $49^{\circ} 54^{\prime} 21^{\prime \prime} \mathrm{w}$ \\
\hline Morretes (PR) & Paraná & Laroca and Mielke (1975) & SPH & $491 \mathrm{~m}$ & $25^{\circ} 26^{\prime} 21^{\prime \prime} \mathrm{s}$ & $48^{\circ} 55^{\prime} 8^{\prime \prime w}$ \\
\hline $\begin{array}{l}\text { Quatro Barras } \\
\text { (PR) }\end{array}$ & Paraná & Laroca et al. (1989) & SPH & $830 \mathrm{~m}$ & $25^{\circ} 26^{\prime} 21^{\prime \prime s}$ & $48^{\circ} 58^{\prime} 47^{\prime \prime} w$ \\
\hline $\begin{array}{l}\text { Serra dos Órgãos } \\
(\mathrm{RJ})^{*}\end{array}$ & Rio de Janeiro & Martin et al. (2011) & SPH & $145 \mathrm{~m} / 2263 \mathrm{~m}$ & $22^{\circ} 29^{\prime} 35^{\prime \prime} \mathrm{s}$ & $43^{\circ} 4^{\prime} 24^{\prime \prime w}$ \\
\hline Uberlândia (MG) & Minas Gerais & Amorim et al. (2009) & SPH & $800 \mathrm{~m}$ & $19^{\circ} 09^{\prime} 20^{\prime \prime s}$ & $48^{\circ} 23^{\prime} 20^{\prime \prime} \mathrm{w}$ \\
\hline Yacutinga (ARG) & Misiones - Argentina & Núñez-Bustos (2008) & SPH & $260 \mathrm{~m}$ & $25^{\circ} 37^{\prime} \mathrm{s}$ & $54^{\circ} 04^{\prime} \mathrm{w}$ \\
\hline
\end{tabular}

*Serra dos Órgãos includes: Cachoeira de Macacu, Guapimirim, Nova Friburgo, Petrópolis and Teresópolis.

**Saturniidae: SAT. Sphingidae: SPH.

86 species in 33 genera of Saturniidae and 377 specimens of 38 species in 15 genera of Sphingidae (Table II). Most species were represented by few individuals concentrated in a group of species (Figure $1 \mathrm{~A}-\mathrm{B})$. Of the 86 species of Saturniidae, 11 species (12.79\%) represented $46.57 \%$ (400) of the individuals and of the 38 species of Sphingidae, 4 species $(10.53 \%)$ represented $45.89 \%$ (173) of the collected specimens. The variation in the relative species abundance of the Saturniidae collected was from $11.17 \%$ to $0.12 \%$ (96 to 1 specimens) and 71 species $(82.56 \%)$ had a relative abundance of less than $2 \%$. For Sphingidae it ranged from $16.44 \%$ to $0.26 \%$ (62 to 1 individuals) and 25 species (65.79 $\%$ ) had a relative abundance of less than $2 \%$.

In Saturniidae, the species which contributed with approximately $50 \%$ of individuals of the total sample of the family were (Table II): Pseudautomeris coronis ( $\mathrm{N}=96 ; \mathrm{RA}=11.21 \%)$, Cerodirphia vagans $(\mathrm{N}=40 ; \mathrm{RA}=4.66 \%)$, Eacles ducalis $\quad(\mathrm{N}=37 ; \quad \mathrm{RA}=4.31 \%), \quad$ Cerodirphia zikani (N=35; $\mathrm{RA}=4.07 \%)$, Dirphia araucariae ( $\mathrm{N}=34$; RA3.96\%), Dirphiopsis epiolina $(\mathrm{N}=31$; $\mathrm{RA}=3.61 \%), \quad$ Mollipa convergens $\quad(\mathrm{N}=31$; $\mathrm{RA}=3.61 \%)$; Hylesia vindex $(\mathrm{N}=27 ; \mathrm{RA}=3.14 \%)$,
Mollipa sabina $(\mathrm{N}=24 ; \mathrm{RA}=2.79 \%)$, Scolesa viettei $(\mathrm{N}=23 ; \mathrm{RA}=2.68 \%)$ and Dirphia muscosa $(\mathrm{N}=22$; $\mathrm{RA}=2.56 \%$ ).

The species of Sphingidae which contributed with approximately $50 \%$ of the samples were (Table II): Erinnyis ello ello ( $\mathrm{N}=62 ; \mathrm{RA}=16.45 \%)$, Adhemarius eurysthenes ( $\mathrm{N}=45 ; \mathrm{RA}=11.94 \%)$, Xylophanes xylobotes $\quad(\mathrm{N}=35 ; \quad \mathrm{RA}=9.28 \%)$ and Xylophanes porcus continentalis $(\mathrm{N}=31$; $\mathrm{RA}=8.22 \%$ ).

Saturniidae has the most extreme differences in abundance due to Pseudautomeris coronis (Schaus, 1913) (96 specimens) that differing in a broad margin from the second most abundant, Cerodirphia vagans (Walker, 1855) (40 specimens) (Table II).

The representativeness of the samples (Figure 2) can be better visualized by observing the estimated richness calculated with Jackknife 2. Saturniidae resulted in 116.24 species which represents $73.98 \%$ of the richness. For Sphingidae, the estimated species richness generated was 50.66 (75.01\% of the observed).

The composition of this assemblage (Table II) indicated that the richest and most abundant 


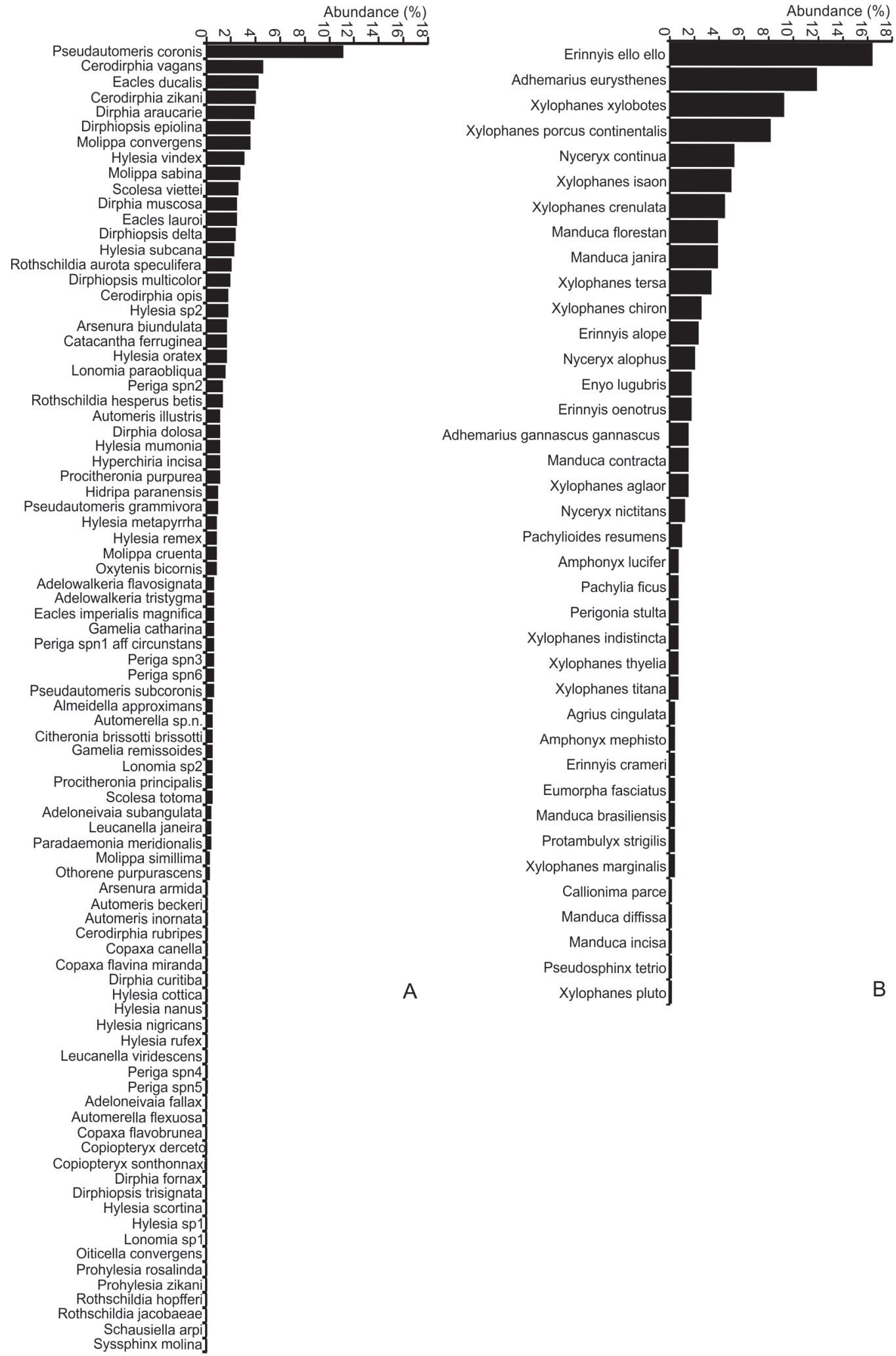

Figure 1 - Curve of the dominance component being represented by the relative abundance of species caught: Saturniidae (A) and Sphingidae (B). 
TABLE II

Checklist of the species of Saturniidae and Sphingidae with their abundance (N) and relative abundance (RA).

SATURNIIDAE

\begin{tabular}{|c|c|c|}
\hline SATURNIIDAE & $\mathbf{N}$ & RA \\
\hline \multicolumn{3}{|l|}{ Arsenurinae } \\
\hline Arsenura armida (Cramer, 1779) & 2 & 0.23 \\
\hline Arsenura biundulata Schaus, 1906 & 15 & 1.75 \\
\hline Copiopteryx derceto (Maassen, [1872]) & 1 & 0.12 \\
\hline Copiopteryx sonthonnaxi É. André, 1905 & 1 & 0.12 \\
\hline Paradaemonia meridionalis Camargo, O. Mielke \& Casagrande, 2007 & 4 & 0.47 \\
\hline Subtotal & 23 & 2.68 \\
\hline \multicolumn{3}{|l|}{ Ceratocampinae } \\
\hline Adeloneivaia fallax (Boisduval, 1872) & 1 & 0.12 \\
\hline Adeloneivaia subangulata (Herrich-Schäffer, [1855]) & 4 & 0.47 \\
\hline Adelowalkeria flavosignata (Walker, 1865) & 6 & 0.70 \\
\hline Adelowalkeria tristygma (Boisduval, 1872) & 6 & 0.70 \\
\hline Almeidella approximans (Schaus, 1921) & 5 & 0.58 \\
\hline Citheronia brissotti brissotti Boisduval, 1868 & 5 & 0.58 \\
\hline Eacles ducalis (Walker, 1855) & 37 & 4.31 \\
\hline Eacles imperialis magnifica Walker, 1855 & 6 & 0.70 \\
\hline Eacles lauroi Oiticica, 1938 & 22 & 2.56 \\
\hline Oiticella convergens (Herrich-Schäffer, [1855]) & 1 & 0.12 \\
\hline Othorene purpurascens (Schaus, 1905) & 3 & 0.35 \\
\hline Procitheronia principalis (Walker, 1855) & 5 & 0.58 \\
\hline Procitheronia purpurea Draudt, 1930 & 10 & 1.16 \\
\hline Schausiella arpi (Schaus, 1892) & 1 & 0.12 \\
\hline Scolesa totoma (Schaus, 1900) & 5 & 0.58 \\
\hline Scolesa viettei Travassos, 1959 & 23 & 2.68 \\
\hline Syssphinx molina (Cramer, 1780) & 1 & 0.12 \\
\hline Subtotal & 141 & 16.41 \\
\hline \multicolumn{3}{|l|}{ Hemileucinae } \\
\hline Automerella flexuosa (R. Felder \& Rogenhofer, 1874) & 1 & 0.12 \\
\hline Automerella sp.n. & 5 & 0.58 \\
\hline Automeris beckeri (Herrich-Schäffer, [1856]) & 2 & 0.23 \\
\hline Automeris illustris (Walker, 1855) & 10 & 1.16 \\
\hline Automeris inornata (Walker, 1855) & 2 & 0.23 \\
\hline Catacantha ferruginea (Draudt, 1929) & 15 & 1.75 \\
\hline Cerodirphia opis (Schaus, 1892) & 16 & 1.86 \\
\hline Cerodirphia rubripes (Draudt, 1930) & 2 & 0.23 \\
\hline Cerodirphia vagans (Walker, 1855) & 40 & 4.66 \\
\hline Cerodirphia zikani (Schaus, 1921) & 35 & 4.07 \\
\hline Dirphia araucariae Jones, 1908 & 34 & 3.96 \\
\hline Dirphia curitiba Draudt, 1930 & 2 & 0.23 \\
\hline Dirphia dolosa Bouvier, 1929 & 10 & 1.16 \\
\hline Dirphia fornax (Druce, 1903) & 1 & 0.12 \\
\hline Dirphia muscosa Schaus, 1898 & 22 & 2.56 \\
\hline Dirphiopsis delta (Foetterle, 1901) & 21 & 2.44 \\
\hline Dirphiopsis epiolina (R. Felder \& Rogenhofer, 1874) & 31 & 3.61 \\
\hline
\end{tabular}




\section{TABLE II (continuation)}

\begin{tabular}{|c|c|c|}
\hline Dirphiopsis multicolor (Walker, 1855) & 17 & 1.98 \\
\hline Dirphiopsis trisignata (R. Felder \& Rogenhofer, 1874) & 1 & 0.12 \\
\hline Gamelia catharina (Draudt, 1929) & 6 & 0.70 \\
\hline Gamelia remissoides Lemaire, 1967 & 5 & 0.58 \\
\hline Hidripa paranensis (Bouvier, 1929) & 9 & 1.05 \\
\hline Hylesia cottica Schaus, 1932 & 2 & 0.23 \\
\hline Hylesia metapyrrha (Walker, 1855) & 8 & 0.93 \\
\hline Hylesia mumonia Schaus, 1927 & 10 & 1.16 \\
\hline Hylesia nanus (Walker, 1855) & 2 & 0.23 \\
\hline Hylesia nigricans (Berg, 1875) & 2 & 0.23 \\
\hline Hylesia oratex Dyar, 1913 & 15 & 1.75 \\
\hline Hylesia remex Dyar, 1913 & 8 & 0.93 \\
\hline Hylesia rufex Draudt, 1929 & 2 & 0.23 \\
\hline Hylesia scortina Draudt, 1929 & 1 & 0.12 \\
\hline Hylesia subcana (Walker, 1855) & 20 & 2.33 \\
\hline Hylesia vindex Dyar, 1913 & 27 & 3.14 \\
\hline Hylesia $\mathrm{sp} 1$ & 1 & 0.12 \\
\hline Hylesia $\mathrm{sp} 2$ & 16 & 1.86 \\
\hline Hyperchiria incisa Walker, 1855 & 10 & 1.16 \\
\hline Leucanella janeira (Westwood, [1854]) & 4 & 0.47 \\
\hline Leucanella viridescens (Walker, 1855) & 2 & 0.23 \\
\hline Lonomia paraobliqua Brechlin, Meister \& Mielke, 2011 & 14 & 1.63 \\
\hline Lonomia obliqua (Walker, 1855) & 5 & 0.58 \\
\hline Lonomia sp1 & 1 & 0.12 \\
\hline Molippa convergens (Walker, 1855) & 31 & 3.61 \\
\hline Molippa cruenta (Walker, 1855) & 8 & 0.93 \\
\hline Molippa sabina Walker, 1855 & 24 & 2.79 \\
\hline Molippa simillima Jones, 1907 & 3 & 0.35 \\
\hline Periga sp1 & 6 & 0.70 \\
\hline Periga sp2 & 12 & 1.40 \\
\hline Periga sp3 & 6 & 0.70 \\
\hline Periga sp4 & 2 & 0.23 \\
\hline Periga sp5 & 2 & 0.23 \\
\hline Periga sp6 & 6 & 0.70 \\
\hline Prohylesia rosalinda Draudt, 1929 & 1 & 0.12 \\
\hline Prohylesia zikani Draudt, 1929 & 1 & 0.12 \\
\hline Pseudautomeris coronis (Schaus, 1913) & 96 & 11.18 \\
\hline Pseudautomeris grammivora (Jones, 1908) & 9 & 1.05 \\
\hline Pseudautomeris subcoronis Lemaire, 1967 & 6 & 0.70 \\
\hline Subtotal & 650 & 75.67 \\
\hline \multicolumn{3}{|l|}{ Oxyteninae } \\
\hline Oxytenis bicornis Jordan, 1924 & 8 & 0.93 \\
\hline Subtotal & 8 & 0.93 \\
\hline \multicolumn{3}{|l|}{ Saturniinae } \\
\hline Copaxa flavobrunnea Bouvier, 1930 & 1 & 0.12 \\
\hline Copaxa canella Walker, 1855 & 2 & 0.23 \\
\hline Copaxa flavina miranda Lemaire, 1971 & 2 & 0.23 \\
\hline
\end{tabular}


TABLE II (continuation)

\begin{tabular}{|c|c|c|}
\hline Rothschildia aurota speculifera (Walker, 1855) & 18 & 2.10 \\
\hline Rothschildia hesperus betis (Walker, 1855) & 12 & 1.40 \\
\hline Rothschildia hopfferi (C. Felder \& R. Felder, 1859) & 1 & 0.12 \\
\hline Rothschildia jacobaeae (Walker, 1855) & 1 & 0.12 \\
\hline Subtotal & 37 & 4.31 \\
\hline Total & 859 & \\
\hline SPHINGIDAE & $\mathbf{N}$ & RA \\
\hline \multicolumn{3}{|l|}{ Macroglossinae } \\
\hline Callionima parce (Fabricius, 1775) & 1 & 0.27 \\
\hline Enyo lugubris (Linnaeus, 1771) & 7 & 1.86 \\
\hline Erinnyis alope (Drury, 1770) & 9 & 2.39 \\
\hline Erinnyis crameri (Schaus, 1898) & 2 & 0.53 \\
\hline Erinnyis ello ello (Linnaeus, 1758) & 62 & 16.45 \\
\hline Erinnyis oenotrus (Cramer, 1782) & 7 & 1.86 \\
\hline Eumorpha fasciatus (Sulzer, 1776) & 2 & 0.53 \\
\hline Nyceryx alophus (Boisduval, [1875]) & 8 & 2.12 \\
\hline Nyceryx continua (Walker, 1856) & 20 & 5.31 \\
\hline Nyceryx nictitans (Boisduval, [1875]) & 5 & 1.33 \\
\hline Pachylia ficus (Linnaeus, 1758) & 3 & 0.80 \\
\hline Pachylioides resumens (Walker, 1856) & 4 & 1.06 \\
\hline Perigonia stulta Herrich-Schäffer, [1854] & 3 & 0.80 \\
\hline Pseudosphinx tetrio (Linnaeus, 1771) & 1 & 0.27 \\
\hline Xylophanes aglaor (Boisduval, [1875]) & 6 & 1.59 \\
\hline Xylophanes chiron (Drury, 1771) & 10 & 2.65 \\
\hline Xylophanes crenulata Vaglia \& Haxaire, 2009 & 17 & 4.51 \\
\hline Xylophanes indistincta Closs, 1915 & 3 & 0.80 \\
\hline Xylophanes isaon (Boisduval, [1875]) & 19 & 5.04 \\
\hline Xylophanes marginalis Clark, 1917 & 2 & 0.53 \\
\hline Xylophanes pluto (Fabricius, 1777) & 1 & 0.27 \\
\hline Xylophanes porcus continentalis Rothschild \& Jordan, 1903 & 31 & 8.22 \\
\hline Xylophanes tersa (Linnaeus, 1771) & 13 & 3.45 \\
\hline Xylophanes thyelia (Linnaeus, 1758) & 3 & 0.80 \\
\hline Xylophanes titana (Druce, 1878) & 3 & 0.80 \\
\hline Xylophanes xylobotes (Burmeister, 1878) & 35 & 9.28 \\
\hline Subtotal & 277 & 73.47 \\
\hline \multicolumn{3}{|l|}{ Smerinthinae } \\
\hline Adhemarius eurysthenes (R. Felder, 1874) & 45 & 11.94 \\
\hline Adhemarius gannascus gannascus (Stoll, 1790) & 6 & 1.59 \\
\hline Protambulyx strigilis (Linnaeus, 1771) & 2 & 0.53 \\
\hline Subtotal & 53 & 14.06 \\
\hline \multicolumn{3}{|l|}{ Sphinginae } \\
\hline Agrius cingulata (Fabricius, 1775) & 2 & 0.53 \\
\hline Amphonyx lucifer (Rothschild \& Jordan, 1903) & 3 & 0.80 \\
\hline Amphonyx mephisto (Haxaire \& Vaglia, 2002) & 2 & 0.53 \\
\hline Manduca brasiliensis (Jordan, 1911) & 2 & 0.53 \\
\hline Manduca contracta (Butler, 1875) & 6 & 1.59 \\
\hline Manduca diffissa (Butler, 1871) & 1 & 0.27 \\
\hline
\end{tabular}


TABLE II (continuation)

\begin{tabular}{lcc}
\hline Manduca florestan (Cramer, 1782) & 15 & 3.98 \\
Manduca incisa (Walker, 1856) & 1 & 0.27 \\
Manduca janira (Jordan, 1911) & 15 & 3.98 \\
\hline Subtotal & $\mathbf{4 7}$ & $\mathbf{1 2 . 4 7}$ \\
\hline Total & $\mathbf{3 7 7}$ & \\
\hline
\end{tabular}

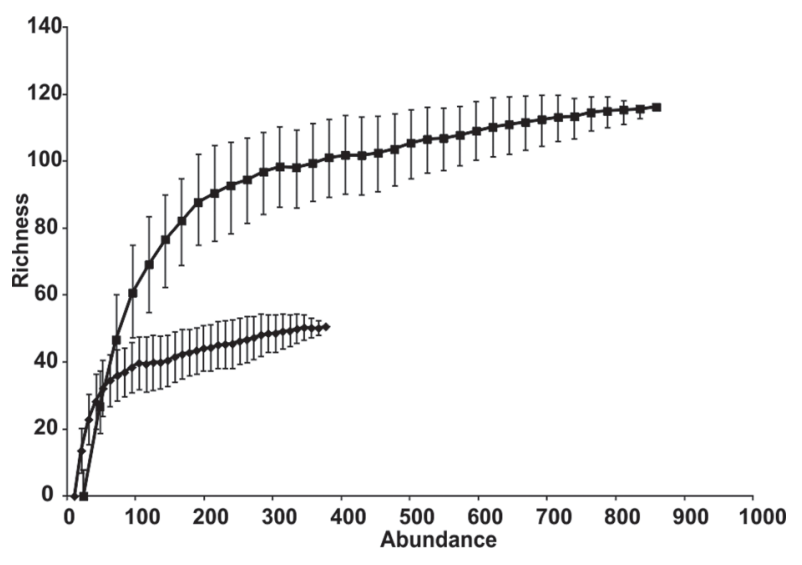

Figure 2 - Curve of estimated richness (Jacknife 2) and their deviations for Saturniidae (points) and Sphingidae (triangles) for 12 samples between November 2010 and September 2011. subfamilies were Hemileucinae (Saturniidae) with 56 species (45.2\%) and 650 individuals (52.6\%) and Macroglossinae (Sphingidae) with 26 species (21\%) and 277 individuals (22.4) (Figure 3 A-B). The abundance follows this same quantitative relationship, with a slight variation in Sphingidae, with Sphinginae being richer than Smerinthinae (Figure 3A) but less abundant (Figure 3B).

Comparing this species richness with that of published inventories in the Paraná, indicated that Tijucas do Sul, has more species of Saturniidae recorded in this state (Figure 4A). In comparison to other localities, it is the fourth largest list, followed by the species lists from Santa Teresa $(\mathrm{S}=123)$,
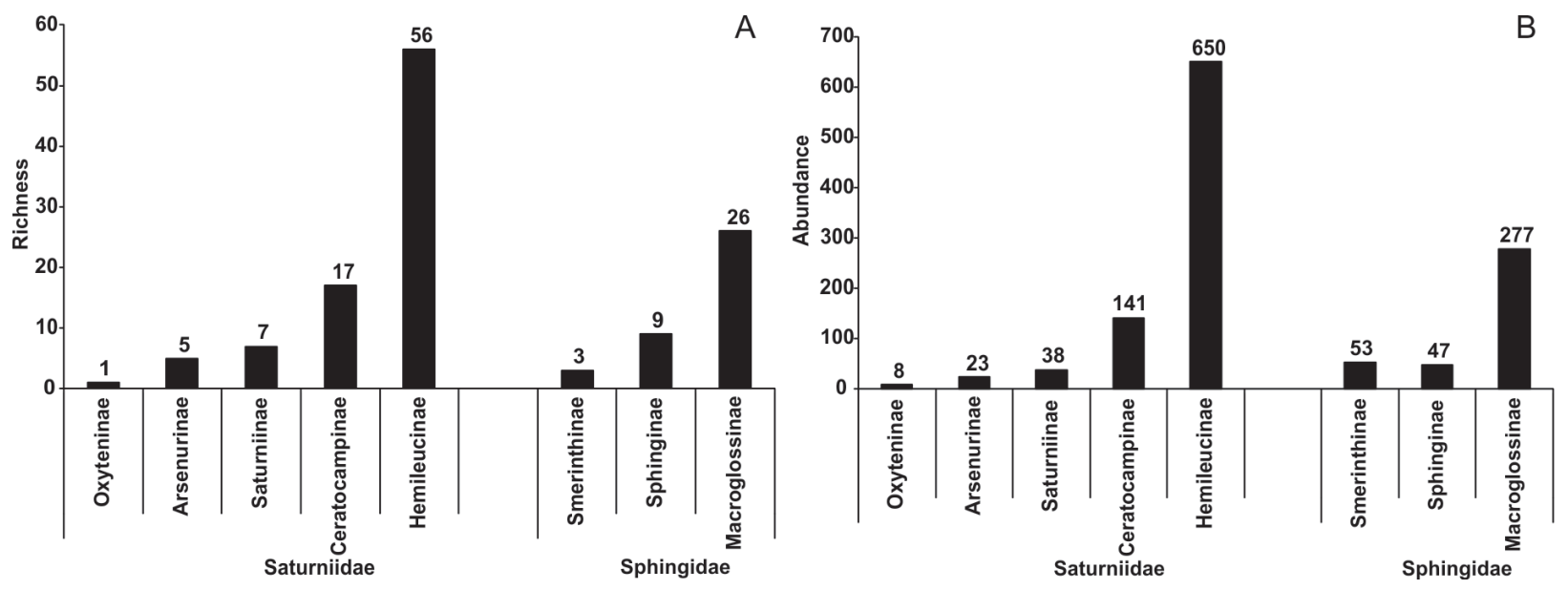

Figure 3 - Species richness (A) and abundance (B) of Saturniidae and Sphingidae by subfamily in Tijucas do Sul, Paraná, Brazil.

São Bento do Sul $(\mathrm{S}=114)$ and Seara $(\mathrm{S}=91)$. In the case of Sphingidae, other localities were richest (Figure 4B). The following locations have highest species richness in the Paraná: Morretes $(S=55)$, Quatro Barras $(\mathrm{S}=51)$ and São José dos Pinhais $(\mathrm{S}=40)$.
In the distribution of richness among the subfamilies of Saturniidae, Hemileucinae (Figure $5 \mathrm{~A}$ ) being the richest, followed by Ceratocampinae (Figure 5B). Hemileucinae tends to appear as the richest subfamily in most localities, especially in lists with a long sampling history, such as that of Santa 

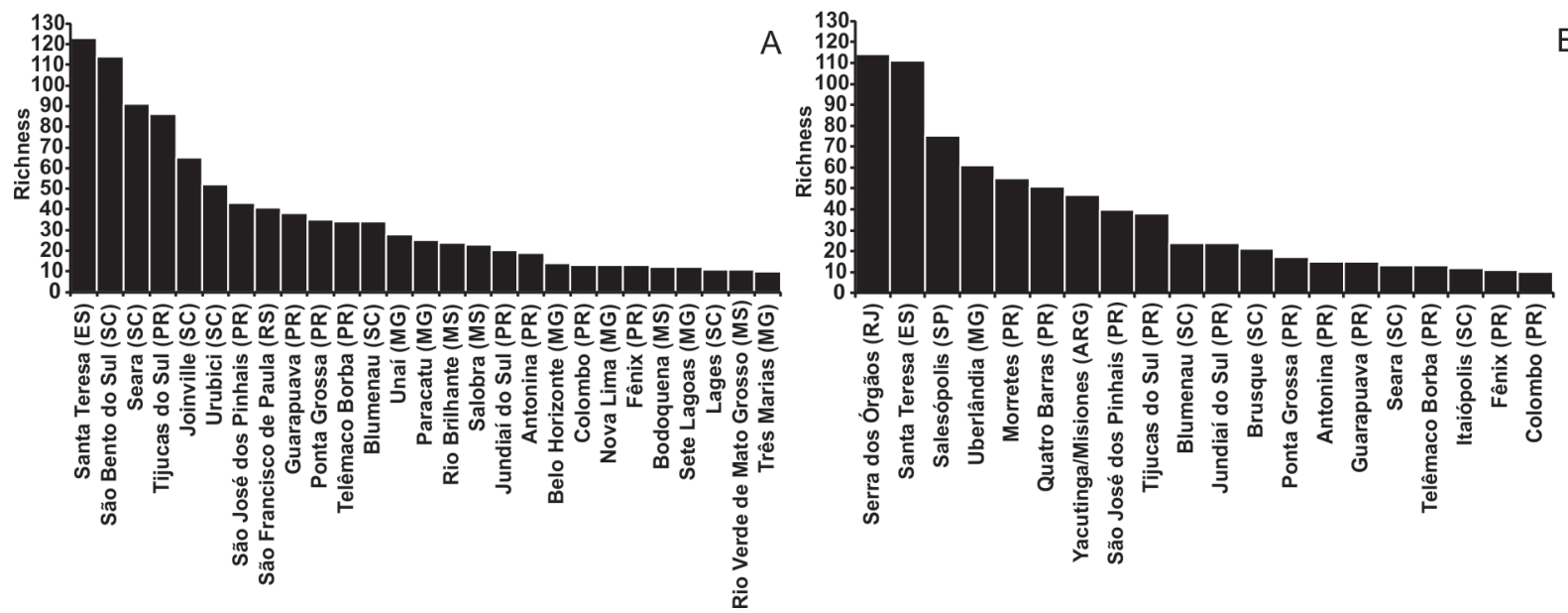

Figure 4 - Richness of Saturniidae (A) and Sphingidae (B) compared between different localities.

Teresa and São Bento do Sul, or with intensified sampling (Figure 5A). With respect to Arsenurinae (Figure 5D), Tijucas do Sul has a richness $(\mathrm{S}=5)$ similar to Guarapuava, Blumenau, Rio Brilhante and Paracatu, and a low richness compared to Santa Teresa $(S=16)$ and São Bento do Sul $(S=13)$. For Saturniinae, with seven species (Figure 5C), it resembles that of Santa Teresa, Joinville and São Francisco de Paula, being less rich than São Bento do Sul and Seara $(S=9)$. Oxyteninae has very few samples and was only present in the lists from Tijucas do Sul and Antonina $(\mathrm{S}=1)$ (Figure 5E).

Macroglossinae was the subfamily of Sphingidae with the greatest number of species and has a large influence on the richness in this inventory (Figure 6A), but when compared with other localities, including the closer areas of Morretes ( $\mathrm{S}=35$ ), Quatro Barras (33) and São José dos Pinhais $(\mathrm{S}=27)$ (Figure $6 \mathrm{~A})$, showed a low richness. With Smerinthinae and Sphinginae this pattern is quite similar (Figure $6 \mathrm{~A}-\mathrm{C}$ ).

Some records of species deserve special attention:

- of the Saturniidae collected species, 14 are recognized as new species to be described: Automerella Michener, 1949 - one species; Hylesia Hübner, [1820] - two species; Lonomia Walker, 1855 - one species; Periga Walker, 1855 - six species.
- the record of Xylophanes crenulata for Paraná does not refer to a new species record. Probably all the old records of Xylophanes ceratomioides from Paraná could be $X$. crenulata (Vaglia and Haxaire 2009).

- Prohylesia rosalinda and Eacles lauroi are new records for Paraná.

- Lonomia obliqua and Lonomia paraobliqua indicated as having different geographic distributions (Brechlin et al. 2011), were collected together in Tijucas do Sul with an overlap of distributions, as well as a short temporal overlap of populations.

\section{DISCUSSION}

This research presents the largest list of Saturniidae published to date for Paraná, with 86 species, including $67.19 \%$ of the 128 Saturniidae species listed for this state of Brazil. The largest previously published list of Marinoni et al. (1997) includes 83 Saturniidae species from eight localities in Paraná, with the greatest richness (43 species) in São José dos Pinhais.

For Sphingidae, 74 species are listed for Paraná, with 51.35\%, 38 species, present in Tijucas do Sul. Unlike the Saturniidae, other geographically close localities have a greater richness of Sphingidae: Morretes (Laroca and Mielke 1975) with 54 species, 


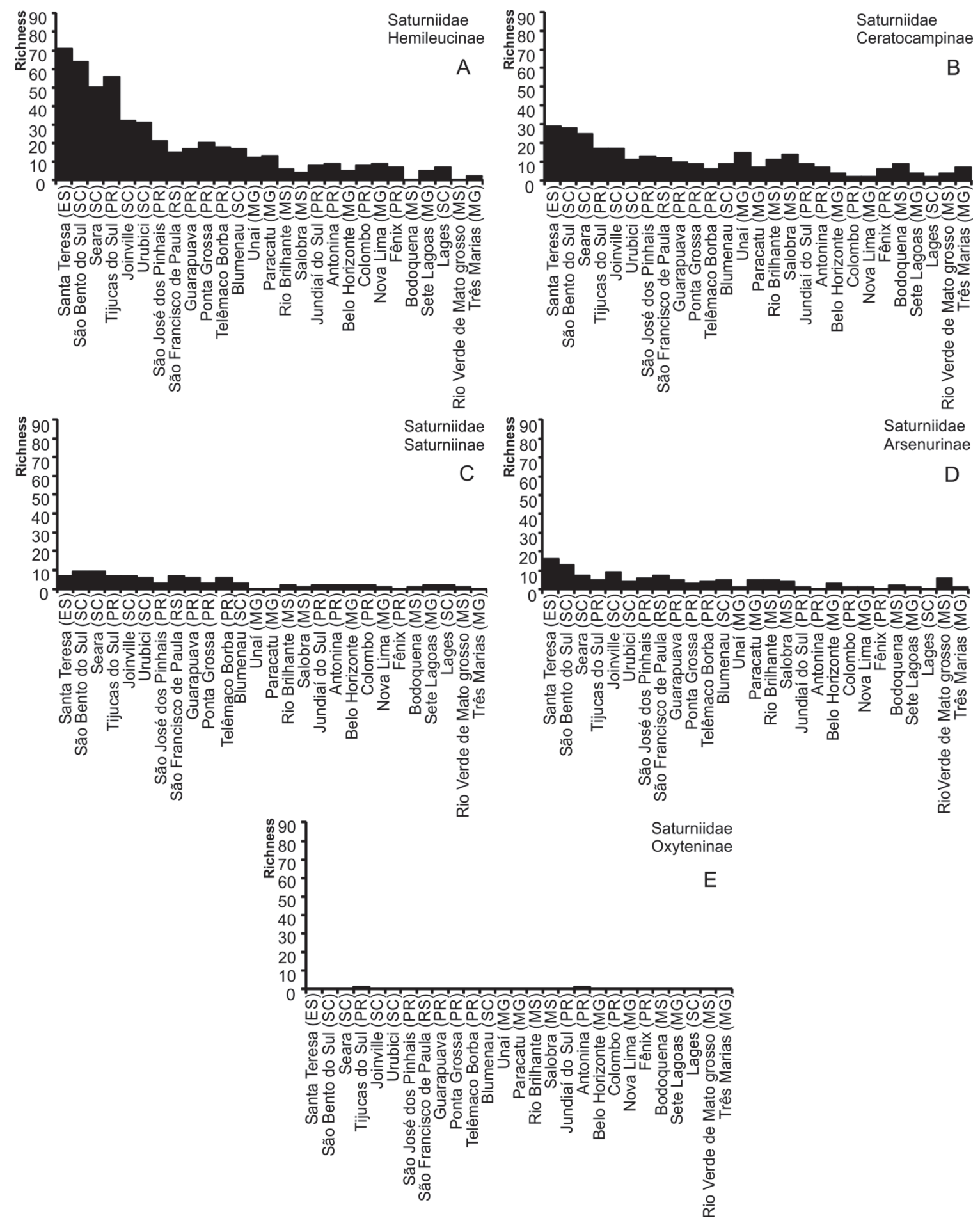

Figure 5 - Richness of the subfamilies of Saturniidae (A-E) compared between different localities.

Quatro Barras (Laroca et al. 1989) with 51 species and São José dos Pinhais with 40 recorded species (Marinoni et al. 1997).
The methodology used in this inventory resulted in a species list which adequately represents the local and regional biodiversity of this assemblage. 


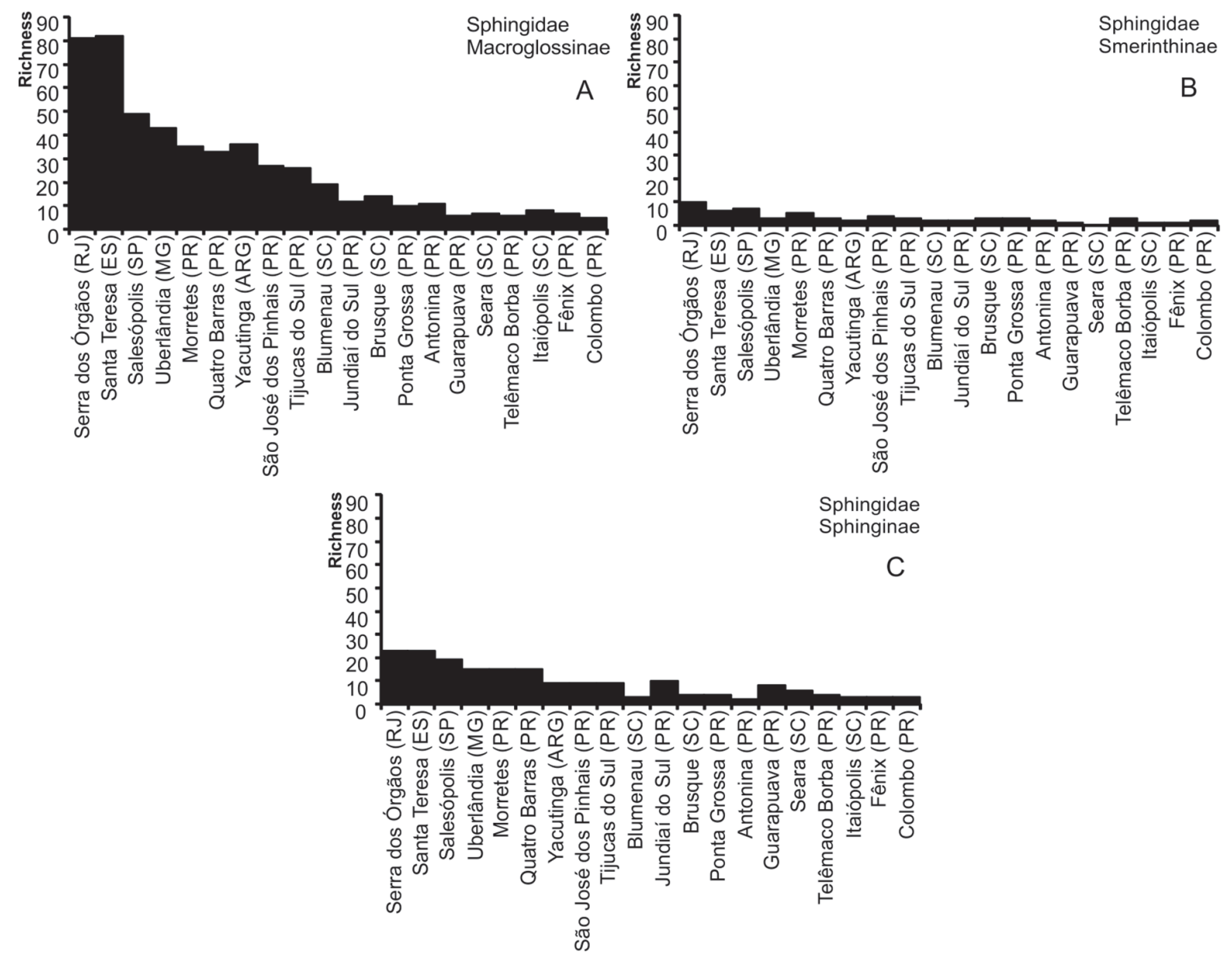

Figure 6 - Richness of the subfamilies of Sphingidae (A-C) compared between different localities.

Furthermore, Tijucas do Sul only has fewer species of Saturniidae when compared to localities where continuous inventories have been conducted for several years and therefore have more complete lists, such as in Santa Teresa, São Bento do Sul and Seara (Figure 4A). The Sphingidae of Tijucas do Sul did not have the same diversity as other nearby areas, which have comparable sampling efforts and similar attraction methods.

The rarefaction curves for the two families indicated that the richness encountered and the expected numbers of species, are greater for Saturniidae than for Sphingidae, considering that the curves and confidence intervals (standard deviation) overlap in the samples under 100 individuals. By analyzing the instability of the species rarefaction curve for Saturniidae, it is possible to infer the existence of species that could be collected and this may suggest the need for additional local or regional collection, with focus on species which have more restricted temporal niches. It is also possible to infer a greater increase of the lists based on the regional diversity presented in other species lists from nearby locations (Marinoni et al. 1997, 1999).

For the Saturniidae, the species richness accumulation curve indicated that further inventories are essential due to the restrictive behavioral characteristics of their adults, which do not feed, have a short seasonal lifespan, a concentrated emergence of individuals, restricted geographical distributions and a reduced migratory capacity. 
The recognition of ten new species was noteworthy, particularly because Tijucas do Sul be close to a research center with a long history of regional collection. With these results it is possible to make forecasts about the expected biodiversity of this assemblage in other areas which remain unknown and are inaccessible to researchers. Furthermore, the number of species, especially those of cryptic genera (i.e. Periga and Hylesia), is expected to increase in areas yet to be sampled, at different times and altitudes, together with the increase of sampling intervals and use of different light sources.

For the subfamily Oxyteninae, there is little information available for comparison as it has been systematically excluded from Saturniidae in some studies, and also because some species are diurnal.

The structure of the Sphingidae community in Tijucas do Sul seems to be very similar to most of the other areas compared herein, especially when compared with areas of similar vegetation structure (i.e. Quatro Barras, Morretes and São José dos Pinhais).

Several characteristics of the Sphingidae may have influenced their low diversity in Tijucas do Sul and the resulting species list. Some of these being: their large dispersal ability and low attractiveness to light traps, already reported for many species (Janzen 1984), their diurnal and / or crepuscular habits, such as in Aellopos (Janzen 1984, Kitching and Cadiou 2000); and the discovery of a few new species, despite the taxonomic stability of the group.

However, with a small increase of the collection effort in Tijucas do Sul, it should be possible to record the species listed in neighboring areas, since local species richness tends to be correlated with regional richness (Caley and Schluter 1997).

The distribution of richness and abundance among the subfamilies generally follows the same quantitative relationship within families, and this can be seen as a general feature for any locality. In general, Hemileucinae and Macroglossinae are the most diverse subfamilies of Sphingidae and Saturniidae, respectively, in the Neotropical region (Heppner 1998). Numerically, Hemileucinae is the most representative of the subfamilies, followed by Ceratocampinae, Saturniinae and Arsenurinae (Lemaire 2002), and Macroglossinae is the most diverse, followed by Sphinginae and Smerinthinae. This pattern appears to be recurrent in the published inventories of these families and does not depend on local habitat (Camargo and Becker 1999, Marinoni et al. 1999). Decaëns et al. (2003) suggest that this pattern in community structure is a representation of the known diversity in each subfamily.

However, even extremely diverse groups may be less rich in certain habitats (Brehm et al. 2003) and further studies should be conducted. For example, Camargo and Becker (1999) suggest that this pattern may be different for the Cerrado biome, suggesting that Arsenurinae and Ceratocampinae tend to be more diversified due to their greater resistance to the water stress of these regions.

In general, one finds a pattern of high dominance for some species and most of the species have low abundances (Magurran 1988). For herbivores this has been a recurring pattern, particularly for moths (Camargo 1999, Novotny and Basset 2000, Teston and Delfina 2010). Both assemblages analyzed in this study have many species with low numbers of individuals, especially evident in Saturniidae. Analyzing the component of dominance, inferred by the slope of the curve formed by the arrangement of relative abundances in descending order, it can be seen that the Saturniidae assemblage has a much more smoother curve than that of Sphingidae, assuming that the more pronounced curvature formed in Sphingidae could indicate lower the evenness (Melo 2008).

The accumulation pattern of individuals per species during the collection period is very similar, even with different abundances, with Saturniidae having high abundances concentrated during shorter periods than those of Sphingidae. In the 
future, the study of the comparative phenology of these groups may allow a broader ecological understanding of how populations of these species are structured over time, in the region.

The most common species of both families differ from those found in other studies, even in those from nearby localities (see Marinoni et al. 1997, 1999). Several factors may be involved in this differentiation, namely temporal factors, with supra-annual events, availability of host plants, and absence or reduction of parasitoids, among others. However, species like Erinnyis ello ello (Sphingidae) are among the most common of the Americas and found in virtually all inventories (Winder 1976).

If we assume that the richness in Tijucas do Sul, Vossoroca, represents the surrounding habitats, it can be inferred that the neighboring localities are very rich for Saturniidae, compared to other inventoried sites located in the Atlantic forest (i.e. São Bento do Sul) and that the regional forest cover is allowing the maintenance of this diversity. As for the Sphingidae community, the composition is very similar to other nearby areas but less rich when compared to places like Morretes and to other inventories with a comparable sampling effort. It is possible that, in this case, for the Sphingidae, the effect of altitude was more important, with a greater richness in lowland areas.

\section{CONCLUSIONS}

Together with the works of Marinoni et al. (1997, 1999), Conte and Machado (2005), Bishop and Scherer-Neto (2010), this study consolidates the region as an area of high species richness. It should be noted that, compared to other studies, the collecting methods used, particularly the sampling effort with uninterrupted collections during the evening and morning twilights, may have been the factor responsible for such a high richness pattern.

The knowledge of the diversity of a specific locality or habitat has been frequently requested, in order to justify policies of preservation, conservation and management. Therefore, as this study shows, knowing the fauna ensures subsidies for conservation and management studies, which are important to develop more efficient strategies, especially in areas that are undergoing rapid degradation as is the situation of the "Floresta Ombrófila Mista Montana" (Lewinsohn et al. 2005, Santos et al. 2008, Nobre et al. 2008, Freitas et al. 2011).

Thus, inventories should be considered as priorities of our society (Conservation International do Brasil et al. 2000, Ministério do Meio Ambiente 2002) due to the increase in the conversion rate of habitats into simplified agro-silvicultural landscapes, which extinguishes more species than the capacity of taxonomists to recognize then (Primack and Rodrigues 2001).

\section{ACKNOWLEDGMENTS}

We are thanklful to Dr. Amabílio Camargo (EMBRAPA/Cerrados), Dr. André Padial (UFPR), M.Sc. Carlos Mielke, M.Sc. Diego Dolibaina (UFPR), Dr. Eduardo Carneiro (UFPR), Dr. Fernando Dias (UFPR), Luziany Queiroz (UFPR), Dr. Maurício Moura (UFPR) e M.Sc. Ricardo Siewert (UFPR) for their help in the identification of species, comments, and the continuous support in field work. The authors are thankful for the critical reviews of anonymous reviewers. They are also grateful to the M.Sc. Roger Hutchings (INPA) for his review and for the English translation. We acknowledge the Associação dos Professores da Universidade Federal do Paraná and their staff members Ademir Velozo and Maria Velozo for granting us permission to access the area and for their invaluable assistance. We extend our gratitude to the Programa de Pós-Graduação em Entomologia (Departamento de Zoologia/UFPR) and to the Conselho Nacional de Desenvolvimento Científico e Tecnológico (CNPq) for the author's fellowships (process N. ${ }^{\circ} 132372 / 2010-0$ ) and for complementary productivity grants. 


\section{RESUMO}

A riqueza e abundância das assembleias de espécies em uma comunidade é uma forma de fundamentar os padrões de diversidade encontrados ao redor do mundo. Este estudo descreve as assembleias de Saturniidae e Sphingidae em uma área de Floresta Ombrófila Mista Montana, Tijucas do Sul, Paraná, Brasil, 880m, 2550'8.93"S, 4902'55.20"W. Amostragens foram coletadas entre Novembro de 2010 e Setembro de 2011 durante duas noites de cada um dos 12 novilúnios do período com armadilha luminosa equipada com lâmpada de luz negra (ultravioleta) de 46 watts entre duas lâmpadas de luz mista de mercúrio HWL 250 watts das 18:00 até as 06:00 h. O esforço amostral totalizou 288 horas, resultando na coleta de 1235 exemplares, representados por 124 espécies, dos quais: 858 exemplares e 86 espécies de Saturniidae e, 377 exemplares e 38 espécies de Sphingidae. Ressaltase a amostragem de 10 novas espécies, sendo uma do gênero de Automerella Michener, 1949; duas de Hylesia Hübner, [1820], uma de Lonomia Walker, 1855 e seis de Periga Walker, 1855 que serão descritas.

Palavras-chave: Neotropical, levantamento, mariposas, comunidade.

\section{REFERENCES}

AMORIM FW, Ávila Jr RS, CAMARGO AJA, VIEIRA AL AND OLIVEIRA PE. 2009. A hawkmoth crossroads? Species richness, seasonality and biogeographical affinities of Sphingidae in a Brazilian Cerrado. J Biogeogr 36: 662-674.

AXMACHER JC AND FIEDLER K. 2004. Manual versus automatic moth sampling at equal light sources - a comparison of catches from Mt. Kilimanjaro. J Lepid Soc 58(4): 194-202.

BALCÁZAR-LARA M AND WOLFE KL. 1997. Cladistics of the Ceratocampinae (Lepidoptera: Saturniidae). Trop Lepid Res 8(Suppl. 2): 1-53.

Balvanera P, Lott E, Segura G, Siebe C And Islas A. 2002. Patterns of $\beta$-diversity in a Mexican tropical dry forest. J Veg Sci 13:145-158.

BISPO AA AND SCHERER-NETO P. 2010. Avian assemblage in a remnant of the Araucaria Forest in the Southeast Parana, Brazil. Biota Neotropica 10(1): 121-130.

BRECHLin R, MEISTER F, MielKe CGC AND VAN SCHAYCK E. 2011. Fünfzehn neue Arten der Gattung Lonomia Walker, 1855 (Lepidoptera: Saturniidae). Entomo-Satsphingia 4(2): 61-77.
BReHM G, SÜSSENBACH D AND FIEDLER K. 2003. Unique elevational diversity patterns of geometrid moths in an Andean montane forest. Ecography 26: 456-466.

BROSE U AND MARTINEZ ND. 2004. Estimating the richness of species with variable mobility. Oikos 105: 292-300.

BROWN Jr KS AND FREITAS AVL. 1999. Lepidoptera. In: BRANDÃO CRF AND CANCELLO EM (Eds), Biodiversidade do Estado de São Paulo, Brasil. Invertebrados Terrestres. São Paulo, FAPESP, p. 225-245.

CALEY MJ AND SCHLUTER D. 1997. The relationship between local and regional diversity. Ecology 78(1): 70-80.

CAMARGO AJA. 1999. Estudo comparativo sobre a composição e a diversidade de lepidópteros noturnos em cinco áreas da Região dos Cerrados. Rev Bras Zool 16(2): 369-380.

CAMARgO AJA AND BeCKER VO. 1999. Saturniidae (Lepidoptera) from the Brazilian Cerrado: composition and biogeographic relationships. Biotropica 31(4): 696705 .

Camargo AJA, Mielke OH And Casagrande MM. 2009. Cladistic analysis of the subfamily Arsenurinae (Lepidoptera, Saturniidae) based on adult morphology. Zootaxa 2218: 1-34.

CARneIro E AND LeIVAS FWT. 2012. Utilizando os hexápodes (Arthropoda, Hexapoda) como bioindicadores na biologia da conservação: avanços e perspectivas. Estud Biol (Curitiba) 34(83): 203-213.

COLWELL RK. 2005. EstimateS: statistic estimation of species richness and shared species from samples. Version 7.5. http://viceroy.eeb.uconn.edu/EstimateS.

CONSERVATION INTERNATIONAL DO BRASIL, FUNDAÇ̃̃O SOS MATA ATLÂNTICA, FUNDAÇÃO BIODIVERSITAS, INSTITUTO DE PESQUisas ECOlÓGICAS, SECRETARIA DO Meio Ambiente do Estado de SÃo PAUlo E Instituto EstADUAL DE FlORESTAS-MG. 2000. Avaliação e ações prioritárias para conservação da biodiversidade da Mata Atlântica e Campos Sulinos. Secretaria de Biodiversidade e Florestas do Ministério do Meio Ambiente. Brasília

CONTE CE AND MACHAdo RA. 2005. Riqueza de espécies e distribuição espacial e temporal em comunidades de anuros (Amphibia, Anura) em uma localidade de Tijucas do Sul, Paraná, Brasil. Rev Bras Zool 22(4): 940-948.

CORDEIRO J AND RODRIGUES WA. 2007. Caracterização fitossociológica de um remanescente de floresta Ombrófila Mista em Guarapuava, PR. Rev Árvore 31(3): 545-554.

D'ABrera B. 1986. Sphingidae Mundi. Hawk Moths of the World. Faringdon: Classey EW Ltd, 256 p.

D'ABrerA B. 1995. Saturniidae Mundi: Saturniidae Moths of the World. Part 1. Keltern: Automeris Press, 177 p.

DeCAËNs T, Bonilla D, RAMIRÉs LD, AMARILlo AR, Wolfe KL, Brosch U AND NAUMANN S. 2003. Diversidad de Saturniidae (Lepidoptera) en la selva andina de San José del Palmar (Alto Chocó, Colombia). Bol Cient Mus Hist Nat U de Caldas (Manizales) 7: 57-75.

Duarte M, Carlin LF and Marconato G. 2008. Lightattracted hawkmoths (Lepidoptera: Sphingidae) of Boracéia, municipality of Salesópolis, state of São Paulo, Brazil. Check List 4(2): 123-136. 
FreitAs AVL ET AL. 2011. Plano de ação nacional para a conservação dos Lepidópteros. In: FREITAS AVL and MARINI-FILHO O (Org), Plano de ação nacional para a conservação dos lepidópteros ameaçados de extinção. Brasilia: ICMBio, p. 1-124.

Galindo-LeAl C AND CÂMARA IG. 2005. Status do hotspot mata atlântica: uma síntese. In: GALINDO-LEAL C AND CÂMARA IG (Eds), Mata Atlântica: biodiversidade, ameaças e perspectivas. São Paulo: Fundação SOS Mata Atlântica, Belo Horizonte: Conservação Internacional, p. 3-11.

GOTELLI NJ AND COLWELL KR. 2001. Quantifying biodiversity: procedures and pitfalls in the measurement and comparison of species richness. Ecol Lett 4: 379-391.

HEPPNER JB (Ed). 1996. Atlas of neotropical Lepidoptera. Checklist: Part4B. Drepanoidea - Bombycoidea Sphingoidea. Gainesville: Scientific Publishers, 87 p.

Heppner JB. 1998. Classification of Lepidoptera. Part 1. Introduction. Holarctic Lepidoptera 5(Suppl. 1): 1-148.

Highland SA, MiLlER JC AND JONES JA. 2013. Determinants of moth diversity and community in a temperate mountainl: vegetation, topography, and seasonality. Ecosphere 4(10): $1-22$.

HIROTA MM. 2005. Monitoramento da cobertura da mata atlântica. In: GALINDO-LEAL C AND CÂMARA IG (Eds), Mata Atlântica: biodiversidade, ameaças e perspectivas. São Paulo: Fundação SOS Mata Atlântica, Belo Horizonte: Conservação Internacional, p. 60-65.

IAP - Instituto Ambiental do PARAnÁ. 2003. Plano de manejo da área de proteção ambiental de Guaratuba. Curitiba: Governo do Estado do Paraná, SEMA, IAP, Diretoria de Biodiversidade e Áreas Protegidas.

IBGE - INSTITUTO BRASILEIRO DE GEOGRAFIA E EsTATísTICA. 1992. Manual técnico da vegetação brasileira: série manuais técnicos em geociências. Rio de Janeiro: Fundação Instituto Brasileiro de Geografia e Estatística.

INMET - Instituto NACiOnAL DE Meteorologia. 2009. Normais climatológicas 1931-1990. www.inmet.gov.br.

JANZEN DH. 1984. Two ways to be a tropical big moth: Santa Rosa saturniids and sphingids. In: DAWKINS R and RIDLEY M (Eds), Oxford Surveys in Evolutionary Biology, V.1. Oxford: Oxford University Press, p. 85-140.

Kawahara AY, Mignault AA, Regier JC, Kitching IJ AND MitTer C. 2009. Phylogeny and biogeography of hawkmoths (Lepidoptera: Sphingidae): evidence from five nuclear genes. Plos One 4(5): e5719. doi: 10.1371/ journal.pone.0005719.

KITCHING IJ AND CADIOU JM. 2000. Hawkmoths of the world: an annotated and illustrated revisionary checklist (Lepidoptera: Sphingidae). Ithaca: Cornell University Press.

LAROCA S, BECKER VO AND ZANELLA FCV. 1989. Diversidade, abundância relativa e fenologia em Sphingidae (Lepidoptera) na Serra do Mar (Quatro Barras, PR), sul do Brasil. Acta Biol Paraná 18(1-4): 13-53.

LAROCA S AND MIELKE OHH. 1975. Ensaios sobre ecologia de comunidades em Sphingidae da Serra do Mar, Paraná, Brasil (Lepidoptera). Rev Bras Biol 35(1): 1-9.
LEMAIRE C. 1978. Les Attacidae américains (=Saturniidae). Attacinae. Neuilly-sur-Seine: Édition C. Lemaire, 238 p.

LEMAIRE C. 1980. Les Attacidae américains (=Saturniidae). Arsenurinae. Neuilly-sur-Seine: Édition C. Lemaire, 199 p.

LEMAIRE C. 1988. Les Attacidae américains (=Attacidae). Ceratocampinae. San José: Museo Nacional de Costa Rica, $480 \mathrm{p}$.

LEMAIRE C. 2002. The Attacidae of America. Hemileucinae. Part A. Keltern: Goecke \& Evers, 688 p.

LEMAIRE C AND Minet J. 1998. The Bombycoidea and their relatives. In: KRISTENSEN NP (Ed), Handbook of zoology. New York: De Gruyter, p. 321-353.

LEWINSOHN TM, FREITAS AVL AND PRADO PI. 2005. Conservação de invertebrados terrestres e seus habitats no Brasil. Megadiversidade 1(1): 62-69.

LIEBSCH D AND ACRA LA. 2004. Riqueza de espécies de subbosque de um fragmento de floresta ombrófila mista em Tijucas do Sul, PR. Ciênc Florest 14(1): 67-76.

MAACK R. 1981. Geografia física do Estado do Paraná. $2^{\text {a }}$ ed., Rio de Janeiro: J. Olympio.

MAGURRAN AE. 1988. Ecological diversity and its measurement, New Jersey: Princeton University Press, 179 p.

MAGURRAN AE. 2004. Measuring biological diversity, Oxford: Blackwell Science, $256 \mathrm{p}$.

MARINONI RC, CASAGRANDE MM AND DUTRA RR. 1997. Levantamento da fauna entomologica do Estado do Paraná. III. Saturniidae (Lepidoptera). Rev Bras Zool 14(2): 473-495.

Marinoni RC, Dutra RR AND Mielke OHH. 1999. Levantamento da fauna entomológica no estado do Paraná. IV. Sphingidae (Lepidoptera). Diversidade alfa e estrutura de comunidade. Rev Bras Zool 16(2): 223-240.

Martin A, SoAres A AND BizArro J. 2011. Guia dos Sphingidae da Serra dos Órgãos, Sudeste do Brasil. Oxford: REGUA Publications.

Melo AS. 2008. O que ganhamos 'confundindo' riqueza de espécies e equabilidade em um índice de diversidade? Biota Neotrop 8(3): 21-27.

MINEROPAR - MiNÉRIOS DO PARANÁ. 2001. Atlas geológico do Estado do Paraná. Curitiba: Universidade Federal do Paraná.

MineT J. 1994. The Bombycoidea: phylogeny and higher classification (Lepidoptera: Glossata). Entomol Scand 25(1): 63-88.

MinistéRIO DO MEIO AMBIENTE. 2002. Biodiversidade brasileira: avaliação e identificação de áreas e ações prioritárias para conservação, utilização sustentável e repartição de benefícios da biodiversidade brasileira. Brasília: MMA/SBF, 404 p.

Mittermeier RA, Myers N, Thomsen JB, Fonseca GB AND OLIVEIRA S. 1998. Biodiversity hotspots and major tropical wilderness areas: approaches to setting conservation priorities. Conserv Biol 12(3): 516-520.

Myers N, MitTermeier RA, MitTermeier GG, FonsecA GAB AND KENT J. 2000. Biodiversity hotspots for conservation priorities. Nature 403(6772): 853-858. 
NEW TR (Ed). 2013. Lepidoptera and invertebrate conservation. In: NEW TR. Lepidoptera and conservation. Oxford: J Wiley \& Sons, Ltd, p. 1-15.

Nobre CEB, Schlindwein C And Mielke OH. 2008. The butterflies (Lepidoptera: Papilionoidea and Hesperioidea) of the Catimbau National Park, Pernambuco, Brazil. Zootaxa 1751: 35-45.

NOVOTNY V AND BASSET Y. 2000. Rare species in communities of tropical insect herbivores: pondering the mystery of singletons. Oikos 89(3): 564-572.

NovOTNY ET AL. 2010. Guild-specific patterns of species richness and host specialization in plant-herbivore food webs from a tropical forest. J Anim Ecol 79(6): 1193-1203.

NunEs FG, PRESTES AS AND CORSEuIL E. 2004. Mariposas do gênero Rothschildia (Lepidoptera, Saturniidae) registradas para o Rio Grande do Sul, Brasil. Biociências 12(1): 31-36.

NunEs FG, Specht A AND CoRseuIL E. 2003. Saturniídeos (Lepidoptera, Saturniidae) ocorrentes no Centro de Pesquisa e Conservação da Natureza Pró-Mata. Divul Mus Ciênc Tecnol - UBEA/PUCRS 8: 55-62.

NúÑEZ-Bustos E. 2008. Las especies de Sphingidae de la Reserva Privada Yacutinga, provincia de Misiones, Argentina (Lepidoptera: Sphingidae). SHILAP Revta lepid 36(142): 219-226.

Prestes AS, Nunes FG, Corseuil E And Moser A. 2009. Arsenurinae and Ceratocampinae (Saturniidae) of Rio Grande do Sul state, Brazil. J Lepid Soc 63(4): 214-232.

PRICE PW. 2002. Resource-driven terrestrial interaction webs. Ecol Res 17:241-247.

PRIMACK R AND RODRIGUES E. 2001. Biologia da conservação. Londrina: Efraim Rodrigues, 328 p.

Regier JC, CoOK CP, Mitter C AND Hussey A. 2008a. A phylogenetic study of the 'bombycoid complex' (Lepidoptera) using five protein-coding nuclear genes, with comments on the problem of macrolepidopteran phylogeny. Syst Entomol 33: 175-189.

Regier JC, Grant MC, Mitter C, COOK CP, Peigler RS AND ROUGERIE R. 2008b. Phylogenetic relationships of wild silkmoths (Lepidoptera: Saturniidae) inferred from four protein-coding nuclear genes. Syst Entomol 33: 219-228.

Ribeiro MC, Metzger JP, Martensen AC, Ponzoni FJ AND HIROTA MM. 2009. The Brazilian Atlantic forest: how much is left, and how is the remaining forest distributed? Implications for conservation. Conserv Biol 142: 1141-1153.
Rondon-Neto RM, Kozera C, ANDRAde RR, CECy AT, HUMMES AP, FritzSONS E, CALDEIRA MVW, MACIEL MNM AND SoUZA MKF. 2002. Caracterização florística e estrutural de um fragmento de Floresta Ombrófila Mista, em Curitiba, PR - Brasil. Floresta (Curitiba) 32(1): 3-16.

RUBINOFF D AND POWELL JA. 2004. Conservation of fragmented small populations: endemic species persistence on California's smallest channel island. Biodivers Conserv 13(13): 25372550.

Santos EC, Mielke OHH And CASAGRAnde MM. 2008. Butterfly inventories in Brazil: the state of art and the priority-areas model research aiming at conservation. Nat conserv 6: 176-198.

SIEWERT RR AND SILVA EJE. 2010. Contribution to the knowledge of the hawkmoths fauna in the state of Santa Catarina, Brazil (Lepidoptera: Sphingidae). Nachr. entomol. Ver. Apollo, NF 31(1/2): 63-66.

SiEwert RR, Silva EJE AND MielKe CGC. 2010. Saturniidae from Santa Catarina state, Brazil, with taxonomic notes (Lepidoptera). Nachr. entomol. Ver. Apollo, NF 30(4): 215-220.

Silveira LF, Beisiegel BM, CURCIO FF, VALduJo PH, Dixo M, Verdade VK, MatToX GMT AND CUNNINGHAM PTM. 2010. Para que servem os inventários de fauna? Estud av (São Paulo) 24(68): 173-207.

Sonego RC, BACKes A AND SouZA AF. 2007. Descrição da estrutura de uma Floresta Ombrófila Mista, RS, Brasil, utilizando estimadores não-paramétricos de riqueza e rarefação de amostras. Acta Bot Bras 21(4): 943-955.

Teston JA And Delfina MC. 2010. Diversidade de Arctiinae (Lepidoptera, Arctiidae) em área alterada em Altamira, Amazônia Oriental, Pará, Brasil. Acta Amaz 40(2): $387-$ 396.

VAGLIA T AND HAXAIRE J. 2009. Description d'un nouveau Sphingidae néotropical, Xylophanes crenulata (Lepidoptera, Sphingidae). The European Entomologist 1(3-4): 95-102.

WINDER JA. 1976. Ecology and control of Erinnyis ello e Erinnyis alope, important insect pests in the New World. PANS 22(4): 449-466. 